\title{
Alteration in Transcriptional State, as a First Step in Cancer Development
}

Uchiumi $\mathbf{F}^{1,2^{*}}$, Larsen $\mathbf{S}^{2}$ and Tanuma $\mathbf{S}^{2,3}$

${ }^{1}$ Department of Gene Regulation, Faculty of Pharmaceutical Sciences, Tokyo University of Science, Noda-shi, Chiba-ken, Japan

${ }^{2}$ Research Center for RNA Science, RIST, Tokyo University of Science, Noda-shi, Chiba-ken, Japan

${ }^{3}$ Biochemistry, Faculty of Pharmaceutical Sciences, Tokyo University of Science, Noda-shi, Chiba-ken, Japan

*Corresponding author: Fumiaki Uchiumi, Department of Gene Regulation, Faculty of Pharmaceutical Sciences, Tokyo University of Science, Tokyo University of Science, 2641 Yamazaki, Noda-shi, Chiba-ken 278-8510, Japan, Tel: +81-4-7121-3616; Fax: +81-4-7121-3608; E-mail: uchiumi@rs.noda.tus.ac.jp

Receiving date: November 04, 2014, Accepted date: November 05, 2014, Published date: December 10, 2014

Copyright: (C) 2014 Uchiumi F, et al. This is an open-access article distributed under the terms of the Creative Commons Attribution License, which permits unrestricted use, distribution, and reproduction in any medium, provided the original author and source are credited.

\section{Abstract}

The risk of cancer, which increases in accordance with aging, has been mainly explained by the oxidative stresses that cause DNA damage. In fact, recent DNA sequencing studies of genomes from cancer patients revealed a variety of mutations on specific genes, suggesting that accumulation of DNA damage is the main cause for development of cancer. Therefore, great effort has been undertaken to analyze DNA mutations. On the other hand, especially for clinical diagnosis, abnormalities in metabolism, including up-regulation of glycolysis or "Warburg effect", are known to be characteristics of cancer. Taken together, cancer could be referred to as both "genetic disease" and a "metabolic disease". We have confirmed that numbers of DNA-repair- and mitochondrial function-associated gene promoters commonly contain a duplicated GGAA-motif, which is a target for multiple transcription factors. In this article, we will tentatively draw a hypothetical mechanism behind the generation of cancerous cells, in which alterations in transcriptional state have occurred primarily with repeated divisions or aging of normal cells. Thus, cancer could be regarded as a "transcriptional disease". We hope this concept contribute for innovation of a new cancer therapeutics targeting transcription.

Keywords: Cancer; DNA repair; Mitochondria; Metabolism; Senescence

\section{Editorial}

It has long been argued how and why cancers are generated. In general, it is believed that cancer is a genetic disease that is resulted from mutations on driver genes, including PIK3CA, IDH1 and RBI [1]. Moreover, next-generation sequencing enabled diagnosis of cancer and diseases that are thought to be occurred from genomic alterations [2]. Another aspect of cancer is that it is a metabolic disease [3]. It is widely known that cancer consumes more glucose to produce ATP by glycolysis or fermentation. The metabolic state of tumor cells has been referred to as the "Warburg effect" [4]. Importantly, TCA-cycle enzymes, FH (Fumarate hydratase) and SDH (Succinate Dehydrogenase) have been suggested as tumor suppressors [5]. Deficiency in oxidative phosphorylation (OxPHOS), which is dependent on an adequate supply of NADH and FADH2 from TCA cycle, has been suggested to play a causative role in cancer development [6,7]. We have confirmed that a cis-element, namely duplicated GGAA motif, is present near transcription start sites of the $C S, A C O 2, I D H 1, I D H 3 A, I D H 3 B, S U C L G 1, S D H A F 2, S D H B, S D H D$, $F H$, and $A C L Y$ genes, which encode enzymes in the TCA-cycle [8]. Moreover, the motif is contained in the bidirectional promoter regions of the genes encoding components of the OxPHOS complex, such as
NDUFA1, NDUFA2, NDUFB3, NDUFB9, NDUFC1, NDUFS1, and NDUFS3 [8].

It is well established that epigenetic and/or transcriptional change play a role to determine chromatin states in tumor cells [9]. Recent genomic studies indicated alterations in gene expression in many human diseases $[10,11]$. Whole transcriptome analysis indicating cisquantitative trait loci (QTLs) has been reported as of value for revealing gene expression and transcription state in cells from patients of specific disease [12]. Cancer incidence in humans increase exponentially with age, suggesting that aging is the strongest demographic risk factor for most human malignancies [13,14]. These events could be explained by reactive oxygen species (ROS) generation and accumulation of DNA damage on chromosomes or increased genomic stability, including telomere shortening [14]. Moreover, hypoxia, which attenuates response to DNA damage, increase mutation rate and chromosomal instability, may play a part in linking cancer with aging $[15,16]$. Importantly, aging is accompanied with epigenetic change and alteration of gene expression profile $[17,18]$. Numbers of GGAA motif-binding transcription factors (TFs), which act as positive and negative transcriptional regulators, may drive mitochondrial- and DNA repair-factor encoding genes simultaneously. However, repeated cell division and extracellular signals will gradually disturb the profile of TFs that bind to the GGAA (TTCC) motifs, and finally lead to disruptions in mitochondrial functions and DNA-repair systems. At this stage, cells will exhibit abnormalities in metabolism carrying mutations on DNAs. These features could be referred to as characteristics of cancer and malignant tumors. Moreover, DNA damage will activate poly(ADP-ribosyl)ation resulting in over consumption of $\mathrm{NAD}^{+}$molecules that are required for synthesis of poly (ADP-ribose). The reduction in $\mathrm{NAD}^{+} / \mathrm{NADH}$ ratio will disturb the progression of the TCA cycle until it does not function for sufficient ATP production. In this way, cells can no longer depend on the normal respiration system, but must up-regulate glycolysis and fermentation (Figure 1).

Thus it might be too late to treat cancer after identification of mutations on driver genes, because they have already abnormalities in DNA repair and mitochondria, which frequently cause chemoresistance. Moreover, most of the anti-cancer drugs have been developed under the concept to kill cancerous cells, but sometimes they have heavy side effects on patients. A novel concept is required to treat pre-malignant cells to be converted into benign state.

It remains unclear how the GGAA motif has been duplicated and incorporated into regulatory regions of various genes encoding DNA repair- and mitochondrial-factors. However, the duplication of TF 
Page 2 of 3

binding site might have been advantageous for organisms in the course of evolution.

The crystal structure of mouse Elf3 with type II TGF- $\beta$ receptor promoter was reported [19], representing an association model of the ets motif binding protein with the duplicated GGAA motifs. The sequence is recognized by at least twenty seven ETS family proteins and other TFs, including GABP, NF- $\mathrm{kB} / \mathrm{c}-\mathrm{Rel}$, and STAT proteins. Therefore, transcriptional efficiency could be fine-tuned with the distance between GGAA (TTCC) sequences, variation of the flanking sequences and the combination of the binding factors.

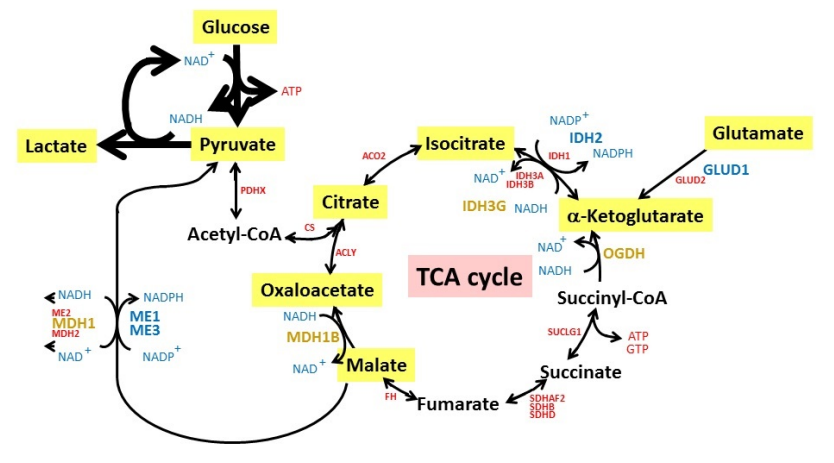

Figure 1: Dysfunction of tricarboxylic acid (TCA) cycle, which could be caused by attenuation of GGAA-dependent transcription. Duplicated GGAA-motifs are contained in the 5'-upstream regions of the ACO2, GLUD2, IDH1, IDH3A,IDH3B, MDH2, ME2, SUCLG1, SDHAF2/SDHB/SDHD, FH, ACLY, CS, and PDHX genes. Various cellular stresses, including chemicals, X-ray and UV irradiation, virus infection, and aging, may alter the transcriptional state leading to disruption of the mitochondrial function- and DNA repair-associated gene expression. Damage on DNAs activate PARP enzyme, which consumes $\mathrm{NAD}^{+}$molecule to synthesize poly(ADPribose), may further lead to dysfunction of the TCA-cycle. Thus cells must depend on glycolysis to synthesize ATP. The consequence could be referred to as the "Warburg effect". This hypothesis implies that pre-cancerous state could be prevented if $\mathrm{NAD}^{+} / \mathrm{NADH}$ ratio were ameliorated.

Here we propose a mechanism in which alterations in the transcriptional state of cells leads to insufficient mitochondrial function and DNA repair system. Given that introduction of OSKM transcription factors reprogram cells to gain pluripotent ability [20], enforced expression of specific TFs may improve metabolism in cancerous cells. In order to access this possibility, elucidation of how human genes, especially those encoding mitochondrial function- and DNA repair-associated factors, are regulated by GGAA motifdependent transcription system. If the mechanism were revealed, novel gene therapy, such as introduction of specific TFs, could be developed to make pre-cancerous cells free from aberrant TCA cycle/respiration with unhealthy ATP-synthesis. In other words, this therapy will upregulate DNA repair system and mitochondrial functions by modulating profile of TFs. We believe that the concept is valuable, though uncultivated as of yet, to find next generation cancer drugs with much lower side effects.

\section{Acknowledgment}

This work was supported in part by JSPS KAKENHI Grant Number 24510270 and a Research Fellowship from the Research Center for RNA Science, RIST, Tokyo University of Science.

\section{References}

1. Vogelstein B, Papadopoulos N, Velculescu VE, Zhou S, Diaz LA, et al. (2013) Cancer genome landscapes. Science 339: 1546-1558.

2. Meyerson M, Gabriel S, Getz G (2010) Advances in understanding cancer genomes through second-generation sequencing. Nat Rev Genet 11: 685-696.

3. Seyfried TN, Flores RE, Poff AM, D'Agostino DP (2014) Cancer as a metabolic disease: implications for novel therapeutics. Carcinogenesis 35: 515-527.

4. Seyfried TN (2012) Chapter 2: Confusion surrounds the origin of cancer. In: Cancer as a metabolic disease. A John Wiley \& Sons, Hoboken, NJ: 15-29.

5. Pollard PJ, Brière JJ, Alam NA, Barwell J, Barclay E, et al. (2005) Accumulation of Krebs cycle intermediates and over-expression of HIFalpha in tumors which result from germline $\mathrm{FH}$ and SDH mutations. Hum Mol Genet 14: 2231-2239.

6. Yadava N, Khalil A, Schneider SS (2013) OxPhos defects and their role in cancer initiation and progression. In: Mitochondria as targets for phytochemicals in cancer prevention and therapy. Springer, New York : $1-26$.

7. Gasparre G, Porcelli AM, Lenaz G, Romeo G (2014) Relevance of mitochondrial genetics and metabolism in cancer development, New York : 235-251.

8. Uchiumi F, Fujikawa M, Miyazaki S, Tanuma S (2013) Implication of bidirectional promoters containing duplicated GGAA motifs of mitochondrial function-associated genes. AIMS Mol Sci 1: 1-26.

9. Suvà ML, Riggi N, Bernstein BE (2013) Epigenetic reprogrammning in cancer. Science 339: 1567-1570.

10. Nicolae DL, Gamazon E, Zhang W, Duan S, Dolan ME, et al .(2010) Traitassociated SNPs are more likely to be eQTLs: annotation to enhance discovery from GWAS. PLoS Genet 6.

11. Nica AC, Montgomery SB, Dimas AS, Stranger BE, Beazley C, et al. (2010) Candidate causal regulatory effects by integration of expression QTLs with complex trait genetic associations. PLoS Genet 6.

12. Lappalainen T, Sammeth M, Friedländer MR, 't Hoen PA, Monlong J, et al. (2013) Transcriptome and genomic sequencing uncovers functional variation in humans. Nature 501: 506-511.

13. Edwards B, Howe HL, Ries LA, Thun MJ, Rosenberg HM, et al. (2002) Annual report to the nation on the status of cancer, 1973-1999, featuring implications of age and aging on U.S. cancer burden. Cancer 94: 2766-2792.

14. Benz CC, Yau C (2008) Aging, oxidative stress and cancer: paradigms in parallax. Nat Rev Cancer 8: 875-879.

15. Bristow RG, Hill RP (2008) Hypoxia, DNA repair and genetic instability. Nat Rev Cancer 8: 180-192.

16. Welford SM, Giaccia AJ (2011) Hypoxia and senescence: the impact of oxygenation on tumor suppression. Mol Cancer Res 9: 538-544.

17. Cencioni C, Spallotta F, Martelli F, Valente S, Mai A, et al. (2013) Oxidative stress and epigenetic regulation in ageing and age-related diseases. Int $\mathrm{J}$ Mol Sci 14: 17643-17663.

18. Geigl JB, Langer S, Barwisch S, Pfleghaar K, Lederer G, et al. (2004) Analysis of gene expression patterns and chromosomal changes associated with aging. Cancer Res 64: 8550-8557.

19. Agarkar VB, Babayeva ND, Wilder PJ, Rizzino A, Tahirov TH (2010) Crystal structure of mouse Elf3 C-terminal DNA-binding domain in 
Citation: Uchiumi F, Larsen S and Tanuma S (2014) Alteration in Transcriptional State, as a First Step in Cancer Development. Pharm Anal Acta 5: e166. doi:10.4172/2153-2435.1000e166

Page 3 of 3

complex with type II TGF- $\beta$ receptor promoter DNA. J Mol Biol 397 278-289.
20. Takahashi K, Yamanaka S (2006) Induction of pluripotent stem cells from mouse embryonic and adult fibroblast cultures by defined factors. Cell 126: 663-676. 